\title{
Linx
}

Revue des linguistes de l'université Paris X Nanterre

$74 \mid 2017$

Claudine Normand, une vie dans le langage

\section{Du signifiant comme structure}

Lacan lecteur de Saussure

\section{Anne-Gaëlle Toutain}

\section{(2) OpenEdition}

Journals

Édition électronique

URL : http://journals.openedition.org/linx/1755

DOI : $10.4000 /$ linx.1755

ISSN : 2118-9692

Éditeur

Presses universitaires de Paris Nanterre

\section{Édition imprimée}

Date de publication : 31 juillet 2017

Pagination : 149-161

ISSN : 0246-8743

Référence électronique

Anne-Gaëlle Toutain, « Du signifiant comme structure », Linx [En ligne], 74 | 2017, mis en ligne le 15 avril 2018, consulté le 19 avril 2019. URL : http://journals.openedition.org/linx/1755; DOI : 10.4000/ linx. 1755

Ce document a été généré automatiquement le 19 avril 2019

Département de Sciences du langage, Université Paris Ouest 


\title{
Du signifiant comme structure
}

\author{
Lacan lecteur de Saussure
}

Anne-Gaëlle Toutain

Claudine Normand s'est beaucoup intéressée à la psychanalyse, en particulier aux rapports de celle-ci avec la linguistique. Je ne saurais donc mieux lui rendre hommage que par cet article consacré à ces derniers, plus précisément à l'utilisation lacanienne de l'œuvre de Saussure.

2 Pourquoi, de nouveau, Lacan, déjà abondamment commenté de ce point de vue, notamment, et de manière remarquablement approfondie et précise, par Arrivé et Milner? Pour deux raisons, liées. En premier lieu, il me semble que ma lecture de Saussure me permet d'envisager sur nouveaux frais l'utilisation lacanienne de Saussure, qu'à la différence d'Arrivé et de Milner je juge fondée sur un malentendu. En second lieu et, comme je m'efforcerai de le montrer, corrélativement, si Lacan est communément considéré comme l'opérateur d'une jonction entre linguistique et psychanalyse, c'est là, selon moi, une conception qui fait obstacle à une meilleure compréhension du langage, au sens du concept bachelardien d'obstacle épistémologique.

\section{D'une étiologie du signe à un mode de signification}

3 Le point nodal de la théorisation saussurienne de la langue est la théorisation du rapport son/sens. Saussure définit la langue comme un système de valeurs purement oppositives, relatives et négatives, définition dans laquelle la notion centrale est celle de négativité, qui implique une rupture avec la connaissance commune : si le donné linguistique est un ensemble d'entités positives, analysables en une partie phonique et une partie sémantique, ces deux parties fussent-elles inséparables, la thèse saussurienne de la négativité des entités linguistiques signifie que ces dernières n'ont cependant d'autre identité que leur existence. La langue saussurienne consiste ainsi, et c'est là la grande nouveauté de cette théorie, non pas en un ensemble de signes, combinaison d'un signifiant et d'un signifié, mais en cette délimitation-combinaison qui est constitutive des signes et qui définit la langue comme «terrain [...] des articulations » (Saussure, 1997 : 
22), c'est-à-dire comme fonctionnement, un fonctionnement dont son et sens, en tant que linguistiques, sont les effets.

Le concept lacanien de signifiant provient de ce que Lacan appelle «l'algorithme saussurien" et qu'il considère comme fondateur de la linguistique ${ }^{1}$. Arrivé a dès longtemps relevé les multiples modifications que Lacan a fait subir au schéma saussurien du signe ${ }^{2}$. Il faut cependant avant tout insister sur le fait qu'il ne s'agit pas chez Saussure d'un « algorithme » ou d'une «formalisation» (Lacan, 1999a : 494), mais d'un corollaire du concept de valeur. La distinction entre signifiant et signifié renvoie à la dualité du signe saussurien, c'est-à-dire, selon une expression de " De l'essence double du langage " (1891), à sa nature de "signe-idée ", par opposition à un signe considéré comme distinct de sa signification ${ }^{3}$. C'est en effet pour éviter le glissement qui fait du signe (entité double au sens d'une dualité constitutive de l'entité même) un signifiant (constituant du signe au sens traditionnel d'assemblage d'un son et d'une idée qui existent et que l'on peut considérer indépendamment l'un de l'autre), que Saussure forgea dans le troisième cours ${ }^{4}$ les termes de signifiant et de signifié, dans la lignée de tentatives précédentes dont témoigne une série de couples terminologiques éphémères ${ }^{5}$. Or, c'est précisément un tel glissement qu'opère la réécriture lacanienne du schéma saussurien du signe, et l'on ne s'étonnera pas, à cet égard, de voir Lacan faire de Saussure le fondateur de la linguistique moderne "dans la diversité des écoles ${ }^{6}$ " (Lacan, 1999a: 494), là où ces écoles - le structuralisme - promeuvent en réalité une linguistique pré-saussurienne ${ }^{7}$. Comme le signale Arrivé 8 , les différentes modifications apportées par Lacan au schéma saussurien du signe sont liées à l'autonomie du signifiant lacanien. En effet, tandis que Saussure pose l'inséparabilité absolue du signifiant et du signifié, constitutive du concept de valeur, Lacan ne s'oppose à la définition traditionnelle du signe que pour définir un autre type de lien entre signifiant et signifié, c'est-à-dire un autre mode de signification, différentiel et fondé sur la suprématie et l'autonomie du signifiant. Au lieu de dualité, il parle ainsi, notamment dans le séminaire sur les Psychoses (1955-1956), de duplicité du signifiant et du signifiés, notion qui implique moins une dualité qu'une distinction, distinction qui fait du signifiant un type de signe. On lit ainsi dans ce séminaire III : "Cela veut dire que le signifiant y a sa cohérence et son caractère propres, qui le distinguent de toute autre espèce de signe.» (Lacan, 1981: 187), où apparait la spécificité de la conception lacanienne du signifiant : comme ordre autonome, ordre générateur de la signification au lieu - conception traditionnelle du signe à laquelle s'oppose Lacan - d'un ensemble de signifiants correspondant à un ensemble de signifiés. Comme on peut le lire dans «L'instance de la lettre dans l'inconscient» $(1957)^{10}$, il faut se déprendre de «l'illusion que le signifiant répond à la fonction de représenter le signifié », "que le signifiant ait à répondre de son existence au titre de quelque signification que ce soit », et mesurer «l'ampleur de $1[\mathrm{a}]$ fonction [des liaisons propres au signifiant] dans la genèse du signifié ». On lira de même dans le séminaire sur Les Psychoses : «Le signifiant ne fait pas que donner l'enveloppe, le récipient de la signification, il la polarise, il la structure, il l'installe dans l'existence. » (Lacan, 1981 : 295-296). C'est là, selon Lacan ${ }^{11}$, « l'intérêt de la considération linguistique du problème ", qui révèle «l'autonomie du signifiant, à savoir qu'il y a des lois qui lui sont propres ".

5 Dans « La psychanalyse et son enseignement » $(1957)^{12}$, le terme de structure renvoie non seulement à la duplicité du signifiant et du signifié, que la structure "noue», mais également au "système signifiant » qui détermine les symptômes. De fait, si certains commentateurs de Lacan ont pu conclure au caractère saussurien de l'emprunt lacanien 
de la notion de signifiant, c'est notamment dans la mesure où le signifiant lacanien est doté d'une propriété essentielle du signifiant (comme du signifié) saussurien: son caractère différentiel. C'est là, en effet, une spécificité du signifiant lacanien comme type de signe, ainsi qu'il apparaît notamment dans le séminaire sur Les Psychoses, où Lacan envisage deux autres types de signes, le signe biologique et la trace, avant d'écrire que « le signifiant est un signe qui renvoie à un autre signe, qui est comme tel structuré pour signifier l'absence d'un autre signe, en d'autres termes pour s'opposer à lui dans un couple » (Lacan, 1981 : 188). Le langage, pour Lacan, « commence à l'opposition » (Lacan, 1981 : 188). Il affirme ainsi dans «L'instance de la lettre dans l'inconscient» (1957) que «[...] la structure du signifiant est, comme on le dit communément du langage, qu'il soit articulé » (Lacan, 1999a: 498). Saussure reprend également la notion traditionnelle d'articulation, mais, comme nous l'avons vu plus haut, pour définir la langue comme «terrain des articulations». Il s'agit en revanche chez Lacan, comme l'annonçait déjà dans le passage du séminaire III le terme de structure, d'une structuration stratifiée du signifiant, celle même qu'ont décrite, avec diverses variations, les structuralistes. Cette propriété de différentialité que le signifiant lacanien partage avec le signifiant saussurien est donc une propriété en trompe-l'œil. Il s'agit là, en effet, de la lecture structuraliste de Saussure, séparant les deux axes (vertical et horizontal) dont la corrélation fait la spécificité du concept de valeur et la rupture saussurienne avec la conception traditionnelle du signe. Chez Lacan, comme chez Hjelmslev, Jakobson ou Benveniste, trois autres linguistes auxquels Lacan se réfère, le langage est rapport son/sens d'une part, structure d'autre part, là où la définition saussurienne de la langue fait de la combinaison le revers de la délimitation, et de la langue un fonctionnement, et non une structure. Aussi Lacan peut-il parler de phonème et de phonologie ${ }^{13}$, là où le concept de valeur implique au contraire le caractère non linguistique de la phonologie ${ }^{14}$. Le sens a beau être redéfini comme un effet ${ }^{15}$, le rapport son/sens demeure comme tel, le sens, et avec lui le rapport son/sens, étant effets de structure, non, comme chez Saussure, de langue.

Cette dualité du rapport son/sens et de la structure, ainsi que la méconnaissance de la perspective étiologique saussurienne, apparaissent de manière tout particulièrement nette lorsque Lacan commente le paragraphe «La langue comme pensée organisée dans la matière phonique » du Cours de linguistique générale ${ }^{16}$. Celui-ci est notamment évoqué dans Les Psychoses ${ }^{17}$, où Lacan pose que "[c]e qui [...] caractérise le langage, c'est le système du signifiant comme tel » puis, évoquant le schéma des deux "flux» dont Saussure illustre son propos, affirme que «M. de Saussure pense que ce qui permet le découpage du signifiant, c'est une certaine corrélation entre signifiant et signifié ", mais que "[c]e schéma est discutable», en raison du glissement de la signification, qui empêche toute correspondance bi-univoque entre signifiant et signifié, ce "glissement incessant du signifié sous le signifiant» (Lacan, 1999a : 499) dont il était question dans "L'instance de la lettre dans l'inconscient ${ }^{18}$ ». Un peu plus loin dans ce séminaire, il sera de même question d'un rapport entre signifiant et signifié qui " parait toujours fluide, toujours prêt à se défaire » (Lacan, $1981: 297)$. Cette « correspondance » entre signifiant et signifié concerne néanmoins chez Saussure un signifiant et un signifié qui se trouvent ainsi constitués comme tels, par l'articulation définitoire de la langue comme fonctionnement et constitutive du son et du sens comme effets de langue. Il s'agit en revanche chez Lacan d'un signifiant et d'un signifié séparables, parce que pris dans un fonctionnement déjà advenu, celui du signifiant comme ordre du symbolique. Il s'agit ainsi, outre d'un langage caractérisé par le système du signifiant, de significations que l'on dira de «discours». Dans le deuxième passage du séminaire III que je viens 
d'évoquer, Lacan substitue d'ailleurs au pôle saussurien de la "pensée » la "masse sentimentale du courant du discours, masse confuse où des unités apparaissent, des îlots, une image, un objet, un sentiment, un cri, un appel» (Lacan, 1981:296), le caractère amorphe de la matière phonique devenant quant à lui « continuité du signifiant » (Lacan, 1981 : 298), c'est-à-dire suspension de l'existence de la phrase à son achèvement. Il en allait de même dans «La chose freudienne ou Sens du retour à Freud en psychanalyse » (1956), où ${ }^{19}$ Lacan opposait le langage comme structure (signifiant) et le discours que constitue le signifié, opposition qui témoigne du caractère descriptif de la théorie lacanienne du signifiant, en regard de la perspective étiologique saussurienne.

7 En réalité, Lacan n'utilise pas la théorie saussurienne de la langue, mais élabore un autre concept, celui du sujet comme effet de langage. Comme il le pose dans « La psychanalyse vraie, et la fausse » $(1958)^{20}$, la découverte freudienne, et le sens du retour à Freud, sont d'avoir reconnu que «ce sont les lois et les effets propres au langage qui [...] constituent la causalité » de l'inconscient, de sorte que «l'action propre du signifiant » «engendr[e] la signification dans le sujet dont elle s'empare, en le marquant comme signifié ». De là vient la définition lacanienne du signifiant comme «ce qui représente le sujet pour un autre signifiant " (Lacan, 1999b : 299), définition qui, comme il apparaît dans Encore (1972-1973), où Lacan affirme que « [n]ous ne connaissons pas d'autre support par où soit introduit dans le monde le Un, si ce n'est le signifiant en tant que tel, c'est-à-dire en tant que nous apprenons à le séparer de ses effets de signifié » (Lacan, 1975b : 48), constitue le véritable enjeu de la « distinction saussurienne » du signifiant et du signifié. C'est donc un autre objet que construit Lacan, et c'est pourquoi, comme le montre Milner ${ }^{21}$ - et d'une autre manière Borch-Jacobsen ${ }^{22}$ - la référence linguistique ne fut qu'un moment de l'élaboration lacanienne, moment structuraliste ou " hyper-structuraliste ». Néanmoins, comme nous l'avons vu ci-dessus, et comme je l'ai montré en détail ailleurs ${ }^{23}$, Saussure n'est pas structuraliste, et il faut donc s'interroger sur les enjeux de cette lecture lacanienne de Saussure, dont, comme nous l'avons vu, le trait principal est non seulement une rupture avec la conception traditionnelle $\mathrm{du}$ signe, mais également une méconnaissance de l'étiologie saussurienne du rapport son/sens.

\section{De l'articulation entre linguistique et psychanalyse}

Arrivé et Milner s'accordent sur l'importance du rôle de Lacan dans l'établissement d'un rapport entre linguistique et psychanalyse. Nous venons néanmoins de voir qu'alors qu'il s'appuie sur Saussure, qu'il considère comme le fondateur de la linguistique moderne, Lacan n'utilise pas la théorisation saussurienne de la langue. Si « la question des rapports entre la psychanalyse et la science du langage se pose dès les premiers travaux de Freud " (Milner, 1997 : 446), Lacan approfondit ces derniers, en montrant la nature langagière de l'inconscient freudien et en définissant le sujet comme effet de langage, à contre-courant de la psychologisation de l'œuvre de Freud qui prévalait à cette époque. Cependant, ce n'est pas là articuler linguistique et psychanalyse, articulation qui consisterait à poser le problème suivant : si l'inconscient est « structuré comme un langage », et si le langage est ainsi un point de convergence entre linguistique et psychanalyse, que signifie et en quoi consiste cette communauté d'objet, et qu'est alors le langage comme objet commun à la linguistique et à la psychanalyse ? Autrement dit, articuler linguistique et psychanalyse consisterait à construire cette communauté d'objet, c'est-à-dire à définir le langage comme objet commun à la linguistique et à la psychanalyse. Or, Lacan fait quant à lui de 
l'inconscient un objet linguistique, redéfinissant ainsi le langage comme un objet psychanalytique, et retirant dès lors toute substance à la question de l'articulation entre linguistique et psychanalyse. Apparait en effet à plusieurs reprises dans ses textes l'idée d'une anticipation par Freud des recherches de Saussure ${ }^{24}$. Plus précisément, pour Lacan, le psychanalyste surpasse le linguiste, qui, comme il apparaît par exemple dans «L'étourdit » $(1972)^{25}$, ne saurait en fait avoir prise sur son objet. Le psychanalyste, en réalité, "fait de la linguistique " (Lacan, 2001: 132), et ce, dès lors, à la place des linguistes, qui ne sauraient cerner la nature du langage, ne serait-ce qu'en raison de leur qualité de «scientifiques ${ }^{26}$ ». Lacan en venait ainsi, dans «Radiophonie» (1970), à cette proposition : «[...] à énoncer que Freud anticipe la linguistique, je dis moins que ce qui s'impose, et qui est la formule que je libère maintenant : l'inconscient est la condition de la linguistique. » (Lacan, $2001:$ 406), à quoi il ajoute un peu plus loin : « L'inconscient peut être comme je le disais la condition de la linguistique. Celle-ci n'en a pas pour autant sur lui la moindre prise.» (Lacan, 2001: 410). Cette proposition se conjugue avec l'appréhension du langage comme «condition de l'inconscient» (Lacan, 2001: 406627). Il faut cependant remarquer son caractère antilacanien, qui tient à ce que manque la définition du langage dont Lacan affirme pourtant qu'il est la condition de l'inconscient et ce comme quoi l'inconscient est structuré. A cette définition se substitue précisément cet objet psychanalytique qu'est l'inconscient structuré comme un langage, et qui, comme psychanalytique, ne peut que nous confronter à la « carence du linguiste » (Lacan, 2001 : p. 410).

9 C'est donc, paradoxalement, d'une part à une élaboration du caractère langagier de l'inconscient, d'autre part à l'impossibilité de toute articulation entre linguistique et psychanalyse, que conduit la théorisation lacanienne, qui apparaît ainsi, pour une part, fondamentalement non lacanienne. Il faut signaler, à cet égard, les difficultés auxquelles se heurte la théorie lacanienne de la psychose, difficultés révélatrices dans la mesure où, comme l'affirme Manier, la psychose nous confronte précisément au problème de la constitution du langage. Cette théorie met notamment en jeu les notions de point de capiton et de métaphore. Dans "Subversion du sujet et dialectique du désir dans l'inconscient freudien » $(1960)^{28}$, le point de capiton est défini comme le moment "par quoi le signifiant arrête le glissement autrement indéfini de la signification ». Lacan le situe alors sur le graphe du désir, où il s'oppose point par point à la métaphore, à laquelle il semble répondre comme un phénomène de discours à un phénomène de langue. Dans le séminaire sur les psychoses, cette notion de point de capiton est d'ailleurs d'abord introduite au cours de l'analyse d'Athalie $e^{29}$. Il y est ensuite ${ }^{30}$ mis en rapport avec le complexe d'ÆEdipe, qui paraît à Lacan un nœud dans la pensée de Freud, ce qu'il explique par le fait que la notion de père donnait à ce dernier « l'élément le plus sensible dans l'expérience de ce qu['il a pour sa part] appelé le point de capiton entre le signifiant et le signifié ». Lacan suggère alors l'existence d'un «nombre minimum de points d'attache fondamentaux entre le signifiant et le signifié nécessaires à ce qu'un être humain soit dit normal, et qui, lorsqu'ils ne sont pas établis, ou qu'ils lâchent, font le psychotique ». Il est néanmoins difficile de voir là autre chose que la description d'un type de discours, étant donné la définition de «Subversion du sujet... » et l'exemple du signifiant crainte dans Athalie. De fait, c'est vers un autre type d'étiologie que s'oriente ensuite $\operatorname{Lacan}^{31}$ : le président Schreber «manque selon toute apparence de ce signifiant fondamental qui s'appelle être père » (Lacan, 1981: 330), ce qui a pour effet, d'une part, que le sujet doit faire avec d'autres signifiants, les "petits chemins ", que l'on est réduit à emprunter 
lorsqu'il n'y a pas de " grand-route » (le signifiant être père), d'autre part, que, au moment où se révèlent déficients les accrochages entre signifiant et signification, le signifiant se met à parler tout seul, comme les « écriteaux » sur le bord du chemin ${ }^{32}$. Lacan entend ici en premier lieu rendre compte du délire du président Schreber ${ }^{33}$. Aussi s'agit-il, sinon de "discours ", du moins de la description d'un type de signifiant, et il est notable, à cet égard, que la métaphore - celle de la grand-route - tienne lieu de théorie. Lacan décrit ici une symbolisation anormale (l'expérience de la couvade) et un discours " dépersonnifié ", sans que l'on sache jamais - c'est là la contradiction de ce double déploiement de la métaphore : "écriteaux» d'une part, "petits chemins » d'autre part - s'il s'agit d'un signifiant "décapitonné » (indépendance du signifiant, parlant tout seul, discours dépersonnifié) ou d'un signifiant manquant au sujet (symbolisation anormale, polarisée par le manque d'un signifiant), décapitonnage et manque d'ailleurs de même significativement exprimés de manière double : du point de vue du sujet (décapitonné ou à qui un signifiant fait défaut), mais surtout en termes de signifiant indépendant ou à quoi rien ne répond chez le sujet ${ }^{34}$. Il s'agit donc tout à la fois d'une structure particulière (manque d'un signifiant) et d'un symptôme (un discours sans signifié, sans sujet, ou délirant), configuration où à un signifiant absent répond un signifiant sans signifié, c'està-dire où, significativement, il ne s'agit de rien d'autre que d'un signifiant : défaillant, délirant, indépendant, mais toujours fonctionnant. On voit ainsi que la notion de signifiant absent, qui vaut pour Lacan étiologie de la psychose, ne s'articule au symptôme que par le biais d'une métaphore, celle de la grand-route, et au prix d'une contradiction. Autrement dit, la perspective lacanienne est de nouveau purement descriptive.

Cette étiologie, qui met en jeu la notion de métaphore, est en outre marquée du même caractère descriptif que lorsqu'il s'agit du point de capiton. Comme nous l'avons vu plus haut, si le point de capiton est "de discours", la métaphore est "de langue». Selon "Subversion du sujet... », la « structure synchronique » qu'est la métaphore " nous porte à l'origine » (Lacan, 1999b : 286), dans la mesure où « s'y constitue l'attribution première [...] par quoi l'enfant d'un seul coup, en déconnectant la chose de son cri, élève le signe à la fonction du signifiant, et la réalité à la sophistique de la signification » (Lacan, 1999b : 286). Concernant la psychose, le rôle étiologique de la métaphore est double. Il s'agit en premier lieu de rendre compte de l'absence du signifiant paternel qui fait la psychose, et que Lacan explique par une "carence de l'effet métaphorique». Dans "D'une question préliminaire à tout traitement possible de la psychose » (1959), évoquant de nouveau une absence du signifiant être père, il distingue en effet entre refoulement (Verdrängung) et «forclusion » (Verwerfung), et définit cette dernière comme « forclusion du signifiant »:

$\mathrm{Au}$ point où, nous verrons comment, est appelé le Nom-du-Père, peut donc répondre dans l'Autre un pur et simple trou, lequel par la carence de l'effet métaphorique provoquera un trou correspondant à la place de la signification phallique. (Lacan, 1999b : 36).

11 S'explique ainsi le déclenchement de la psychose. Celle-ci, comme dans le cas du président Schreber ${ }^{35}$, se déclenche lorsque le « Nom-du-Père, verworfen, forclos, c'est-àdire jamais venu à la place de l'Autre, y [est] appelé en opposition symbolique au sujet » (Lacan, 1999b: 55). Les choses sont plus compliquées lorsqu'il s'agit de la structure psychotique, que Lacan définit comme un accident du registre du signifiant, mais sans néanmoins que nous soient donnés les moyens de comprendre les raisons de cet accident - sinon par le trou de l'Autre, c'est-à-dire dans le cadre d'une explication dont la circularité est dénoncée par Manier ${ }^{36}$. On lit en effet également dans «D'une question préliminaire à tout traitement possible de la psychose » : 
C'est dans un accident de ce registre et de ce qui s'y accomplit, à savoir la forclusion du Nom-du-Père à la place de l'Autre, et dans l'échec de la métaphore paternelle que nous désignons le défaut qui donne à la psychose sa condition essentielle, avec la structure qui la sépare de la névrose. (Lacan, 1999b : 53).

Ce registre est celui la chaîne signifiante, inaugurée par la symbolisation primordiale. Or, cette dernière, telle que la conçoit Lacan ${ }^{37}$, renvoie à la prise du sujet dans le symbolique, c'est-à-dire à l'avènement du signifiant comme signification du sujet, mais sa notion ne donne aucune indication sur ce qu'implique - d'autre que son résultat : le fonctionnement du signifiant - cette entrée dans le langage, ses modalités, ses caractéristiques, et donc sur la manière dont un tel « accident » pourrait se produire. Une fois de plus, Lacan décrit ici un type de signifiant, dont le fonctionnement est polarisé par l'absence d'un signifiant, et qui implique d'ailleurs lui aussi le fonctionnement de la métaphore, plus précisément d'une « métaphore délirante » (Lacan, 1999b : 55). C'est donc, sous le nom de théorie de la psychose, la description d'un symptôme que nous propose Lacan. Plus précisément, il faut insister de nouveau sur le caractère antilacanien de cette élaboration théorique. Comme le souligne Borch-Jacobsen ${ }^{38}$, l'autonomie du signifiant n'implique en aucun cas l'indépendance du signifié ; c'est là, précisément, le sens du signifiant comme type de signe. Aussi est-il extrêmement notable que sa théorisation de la psychose conduise Lacan à s'intéresser à la jonction du signifiant et du signifié. Cette recherche, en effet, ne peut que le reconduire à la définition traditionnelle du signe, définition contre laquelle il s'inscrit par ailleurs en faux, et qui implique ainsi, paradoxalement, une position antilacanienne. Antilacanienne et, ajouterai-je, présaussurienne, et c'est pourquoi la rupture que constitue la théorie de Manier est nécessairement double : rupture avec la théorisation lacanienne de la psychose, et rupture avec la lecture lacanienne de Saussure, double rupture dont l'un des effets est en outre, tout aussi nécessairement, de donner sa pleine mesure à la théorisation lacanienne du sujet comme effet de langage.

Devient par ailleurs possible, dans ce cadre, d'articuler linguistique et psychanalyse, c'està-dire, outre, comme l'a fait Manier, d'utiliser la théorisation saussurienne de la langue pour rendre compte du langage et du locuteur, articulation de la psychanalyse sur la linguistique, de réfléchir à la nature du langage comme objet commun à la linguistique et à la psychanalyse. A cet égard, il faut rappeler, pour conclure, la position de Milner concernant les rapports entre linguistique et psychanalyse. En effet, si pour Milner ${ }^{39}$, comme pour Lacan, lalangue est le revers de la langue, qui n'a rien à en dire, sinon en attestant de la possibilité d'une formalisation, c'est dans la mesure où la conception milnerienne de la langue est idiomologique. Or, le concept saussurien de valeur, qui définit la langue comme un fonctionnement dont les unités linguistiques (le donné idiomologique) sont l'effet, implique précisément une distinction entre langue et idiome, c'est-à-dire la promotion de la langue comme objet de la linguistique, ouvrant l'espace du langage comme espace de théorisation. 


\section{BIBLIOGRAPHIE}

ARRIVÉ, M., 1987, Linguistique et psychanalyse, Paris, Méridiens/Klincksieck.

- 1994 [2005], Langage et psychanalyse, linguistique et inconscient, Limoges, Lambert-Lucas.

BORCH-JACOBSEN, M., 1990 [1995], Lacan, Paris, Flammarion.

LACAN, J., 1975a, Les Écrits techniques de Freud, Paris, Seuil.

- 1975b, Encore, Paris, Seuil.

- 1981, Les Psychoses, Paris, Seuil.

- 1999a, Écrits I, Paris, Seuil.

- 1999b, Écrits II, Paris, Seuil.

- 2001, Autres écrits, Paris, Seuil.

MANIER, A., 1995, Le Jour où l'espace a coupé le temps. Étiologie et clinique de la psychose, Plancoët, La Tempérance.

MILNER, J.-C., 1978, L'Amour de la langue, Paris, Seuil.

- 1997, « Linguistique », in Dictionnaire de la psychanalyse, Paris, Albin Michel, p. 441-453.

- 2002, Le Périple structural. Figures et paradigme, Paris, Seuil.

NORMAND, C., 2001, «Linguistique et/ou psychanalyse : de leur relation si elle existe », in Arrivé, M. \& Normand, C. (éds.), Linguistique et psychanalyse, Paris, In Press, p. 17-30.

SAUSSURE, F. (de), 1997, Deuxième Cours de linguistique générale (1908-1909), Oxford, New York, Tokyo, Pergamon.

- Écrits de linguistique générale, Paris, Gallimard.

- 2005, « Ferdinand de Saussure : Notes préparatoires pour le cours de linguistique générale 1910-1911, Emile Constantin : Linguistique générale. Cours de M. le professeur de Saussure 1910-1911 », Cahiers Ferdinand de Saussure, 58, Genève, Droz, p. 83-289.

TOUTAIN, A.-G., 2012, « Montrer au linguiste ce qu'il fait. » Une analyse épistémologique du structuralisme européen (Hjelmslev, Jakobson, Martinet, Benveniste) dans sa filiation saussurienne, Paris IV, Thèse de doctorat. Disponible sur : http://tel.archives-ouvertes.fr/tel-00788676.

ToUTAIN, A.-G., 2014, La rupture saussurienne. L'espace du langage, Louvain-la-Neuve, AcademiaBruylant.

ToUTAIN, A.-G., 2015, La problématique phonologique. Du structuralisme linguistique comme idéologie scientifique, Paris, Classiques Garnier.

\section{NOTES}

1. Voir notamment Lacan, 1999a : 494.

2. Voir Arrivé, 1987 : 135-136 et Arrivé, 1994 : 87-88. 
3. Voir Saussure, $2002: 20$ et 20-21.

4. Voir Saussure, $2005: 237-238$.

5. Voir notamment Saussure $2002: 113$ et 258 , à quoi répond dans le troisième cours Saussure $2005: 221$

6. Ces assimilations de Saussure au structuralisme sont nombreuses dans les textes de Lacan. Voir Toutain 2012.

7. Voir Toutain 2012.

8. Voir Arrivé, 1987 : 135-136.

9. Voir également Lacan, $1981: 136,187,195$ et 200.

10. Voir Lacan, 1999a : 494-495.

11. Voir Lacan, $1981: 223$.

12. Voir Lacan, 1999a : 441-442.

13. Voir Lacan, 1999a : 498.

14. Dans la mesure où le son ne saurait être constitutif de la langue qu'en tant que signe, l'objet de la phonologie ne saurait être linguistique. Voir notamment Saussure, $2002: 20-21$ et 202 ainsi que $2005: 223-224$.

15. Voir dans "Radiophonie" (1970), où Lacan entend n'« admett[re] de sens qu'à ce que le réseau en réponde, et de l'incidence d'un effet, oui, - d'un contenu, non » (Lacan, 2001 : 404).

16. Voir Saussure, $1972: 155-157$.

17. Voir Lacan, $1981: 135$.

18. Où Lacan mentionnait déjà cette «image qui ressemble aux deux sinuosités des Eaux supérieures et inférieures dans les miniatures des manuscrits de la Genèse » (Lacan, 1999a : 499-500).

19. Voir Lacan, 1999a : 411-412.

20. Voir Lacan, $2001: 166$.

21. Voir Milner, 2002 : 142-150 et Milner, $1997:$ 448-451.

22. Voir Borch-Jacobsen, 1990 : 207-231, où l'auteur démontre que la théorie saussurienne de la langue chez Lacan ne fait que venir nourrir une philosophie du sujet qui lui préexiste.

23. Voir notamment Toutain 2014 et 2015

24. Voir par exemple Lacan, 1999a : 144 et $2001: 166-167$.

25. Voir Lacan, 2001 : 489. Voir également Lacan, 1999a : 465.

26. Voir notamment Lacan, 1975b : 175-176 et Lacan, $2001:$ 410-411.

27. Voir également à cet égard Lacan, $2001: 488$.

28. Voir Lacan, 1999b : 285-286.

29. Voir Lacan, 1981 : 303-304.

30. Voir Lacan, 1981 : 304.

31. Qui affirme d'ailleurs significativement quelques lignes plus bas : «Je ne vous dis pas que c'est là le ressort du mécanisme de la psychose, je dis que le mécanisme de la psychose s'y manifeste. » (Lacan, 1981 : 305).

32. Voir Lacan, 1981 : 326 sqq., notamment 329-331.

33. Voir à la fin de ce développement : Lacan, 1981 : 331.

34. Voir Lacan, 1981 : 315 et 318 puis 319-320.

35. Voir Lacan, $1981: 320$, mentionné dans la note précédente.

36. Voir Manier, 1995 : 77-78.

37. Voir notamment Lacan, $1981: 169-170,1999 a: 317$ et $2001: 162-164$.

38. Voir Borch-Jacobsen, $1990: 220-221$.

39. Voir Milner, $1997:$ 452-453, $2002: 150-151$ et surtout 1978, notamment 7-9, 20-29 et 65-69. 


\section{RÉSUMÉS}

Si Lacan est communément considéré comme l'opérateur d'une jonction entre linguistique et psychanalyse, une telle représentation fait obstacle - au sens du concept bachelardien d'obstacle épistémologique - à une meilleure compréhension du langage. La lecture lacanienne de Saussure est en effet fondée sur un malentendu : comme les linguistes structuralistes, Lacan disjoint les deux axes du concept saussurien de valeur. La référence lacanienne à Saussure aboutit ainsi paradoxalement à une proposition antilacanienne: au lieu de s'interroger sur la nature du langage comme objet commun à la linguistique et à la psychanalyse, Lacan fait de l'inconscient freudien un objet linguistique, redéfinissant ainsi le langage comme objet psychanalytique et retirant par là même toute substance à la question de l'articulation entre linguistique et psychanalyse.

\section{INDEX}

Mots-clés : signifiant, langage, valeur, psychose, structure

\section{AUTEUR}

\section{ANNE-GAËLLE TOUTAIN}

Institut de langue et de littérature françaises de l'université de Berne (Suisse) \& Laboratoire « Histoire des théories linguistiques » (UMR 7597, Paris) 